\title{
FORMES HISTOLOGIQUES PARTICULIERES DU CANCER DU NASOPHARYNX
}

\author{
H. DHOUIB, M. MNEJJA, W. BOUAYED, A.CHAKROUN, T. BOUDAWARA*, \\ I. CHARFEDDINE, M. FRIKHA**, J. DAOUED***, A. GHORBEL, \\ SERVICE ORL \\ *SERVICE D'ANATOMOPATHOLOGIE \\ **SERVICE DE CARCINOLOGIE MÉDICALE \\ ***SERVICE DE RADIOTHÉRAPIE \\ HÔPITAL HABIB BOURGUIBA, SFAX
}

\begin{abstract}
- RESUME
Le carcinome épidermoïde du nasopharynx est la forme histopathologique la plus fréquente. Cependant, d'autres formes histologiques, bien qu'exceptionnelles peuvent se voir. Le but de notre travail est d'étudier le profil thérapeutique et évolutif de ces entités.

Nous rapportons trois observations particulières de cancer du cavum. II s'agissait de 3 patientes âgées respectivement de 15, 36 et 33 ans. La première patiente, atteinte d'un mélanome malin du cavum (T2aN2Mo) (UICCC 97), a bénéficié d'un évidement ganglionnaire cervical radical droit suivi d'une chimiothérapie. La patiente est décédée un mois et demi plus tard.

La deuxième patiente, atteinte d'un carcinome mucoépidermoïde du cavum (T4NoMo) (UICCC 97) a bénéficié d'une radio-chimiothérapie concomitante avec rémission complète à 17 mois de recul.

La troisième patiente, présente un carcinome adénoïde kystique du cavum (T2bNoMo) (UICCC 97), a bénéficié d'une exérèse tumorale large avec évidement ganglionnaire cervical homolatéral suivie d'une radiothérapie post opératoire. Elle a présenté des métastases pulmonaires 12 mois après, traitées par chimiothérapie avec une réponse partielle. Actuellement, elle est à 18 mois de recul après la chimiothérapie.

Ces formes histopathologiques du cancer du cavum sont exceptionnelles ( $<1 \%$ des tumeurs malignes du cavum). II n'existe aucune particularité clinique ou radiologique et le diagnostic reste histopathologique.

Ces tumeurs posent un problème de prise en charge puisqu'il n'existe pas de traitement codifié pour ces formes rares. Le pronostic dépend du stade de la maladie et du type histologique.

Mots clés : nasopharynx, cancer, mélanome, carcinome mucoépidermoïde, carcinome adénoïde kystique, chirurgie, radiothérapie, chimiothérapie.
\end{abstract}

The nasopharyngeal epidemoid carcinoma is the most frequent histopathological form. However other histological forms can be seen.

The aim was to study therapeutic features and progressive profile of these entities.

We report three particular cases of nasopharyngeal cancer. The patients are females aged respectively 15, 36 and 33 years. The first patient, suffering from malignant nasopharyngeal melanoma (T2a N2 M0) (UICCC 97), has benefited from right radical neck dissection followed by chemotherapy. She died a month and a half after the treatment.

The second patient, suffering from nasopharyngeal mucoepidemoid carcinoma (T4NOMO) (UICCC 97) has benefited from a concomitant radio-chemotherapy with a total recovery after a 17 month treatment.

The third patient, suffering from cystic adenoid carcinoma of the nasopharynx (T2bNOM0) (UICCC 97) has underwent a large tumors resection with homolateral neck dissection followed by post operative radiotherapy. The patient presented lung metastases after a 12 months period treated with chemotherapy. Actually she is at 18 month after chemotherapy with persistence of lung metastasis.

These histopathological forms of nasopharyngeal cancer are exceptional $(<1 \%$ of nasopharyngeal malignant tumors). There are no special clinical or radiological features. The diagnostic is mainly histopathologic. These tumors create a huge treatment difficulty. In fact, there isn't a codified treatment for these rare forms. The prognostic depends basically on the level of the sickness and the histological type.

Keys words : nasopharynx, cancer, melanoma, mucoépidermoïd carcinoma, adénoïd cystic carcinoma, surgery, radiotherapy, chimiotherapy.

\section{INTRODUCTION}

Le cancer du cavum occupe une place à part dans l'ensemble des cancers des voies aérodigestives supérieures vu ses particularités épidémiologiques. Le carcinome épidermoïde est la forme histologique la plus fréquente. Cependant, d'autres formes, bien que rares, peuvent se voir.
Nous rapportons 3 observations de formes anatomopathologiques particulières du cancer du cavum : un mélanome, un carcinome mucoépidermoïde et un carcinome adénoïde kystique.

Nous avons étudié le profil thérapeutique et évolutif de ces formes rares. 


\section{Observation 1 :}

Mlle I. N. âgée de 15 ans, sans antécédents particuliers, a consulté en Août 2003 pour des tuméfactions cervicales bilatérales évoluant depuis 1 mois, avec un syndrome rhinologique et otologique droits, dans un contexte d'altération de l'état général. L'examen cervical a montré un magma d'adénopathies jugulo-carotidien bilatéral mesurant $3 \mathrm{~cm}$ de diamètre à droite et de $2 \mathrm{~cm}$ à gauche. L'examen par nasofibroscope a objectivé une tumeur ulcéro-bourgeonnante comblant le cavum arrivant aux deux choanes. La TDM et l'IRM ont conclu à un processus infiltrant de la paroi latérale droite du cavum ( fig 1 et 2).

L'examen anatomopathologique sur biopsie profonde du cavum a montré, après étude immunohistochimique l'aspect d'un mélanome malin du cavum ( fig 3 et 4). Une enquête étiologique a été alors réalisée comportant :

- un examen cutané complet ayant objectivé 3 lésions papuleuses noirâtres au niveau de la nuque, du dos et de l'avant bras dont les biopsies exérèses étaient en faveur d'un nævus congénital.

- Un examen ophtalmologique, à la recherche d'une tumeur rétinienne, s'est révélé normal.

Le bilan d'extension locorégional et à distance a permis de classer la tumeur T2a N2M0 (UICC 97).

Le protocole thérapeutique a comporté : un évidement ganglionnaire fonctionnel bilatéral puis une chimio-radiothérapie. En peropératoire, la découverte d'adénopathies adhérentes à l'axe vasculaire a imposé un curage radical droit. La chirurgie à gauche était alors impossible compte tenu du caractère infiltrant des adénopathies. La chimiothérapie, à base de Déticène $(800 \mathrm{mg} / \mathrm{m} 2)+$ Vélbé $(1,6$ $\mathrm{mg} / \mathrm{m} 2)+$ cisplatyl, $(20 \mathrm{mg} / \mathrm{m} 2)$ a été réalisée en 6 cures. Au cours de la première cure (9ème jour), la patiente a présenté un déficit de l'hémicorps droit en rapport avec une compression de la carotide droite par un magma d'adénopathies récidivantes. Après six cures de chimiothérapie, la patiente était en poursuite évolutive locale et ganglionnaire massive.

Elle est décédée un mois et demi plus tard.

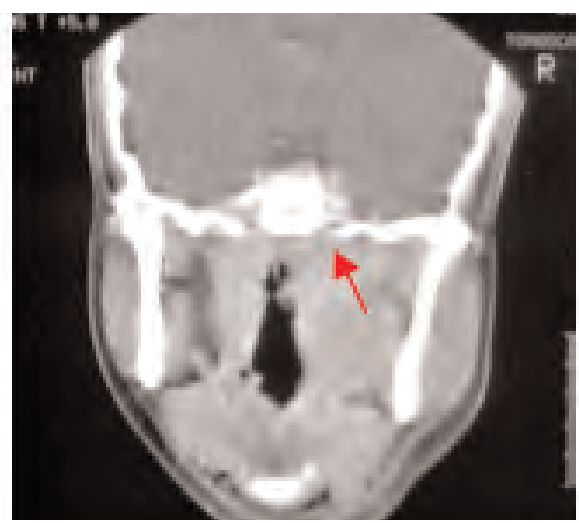

Fig.1 : TDM en coupe frontale avec injection : Tumeur du cavum envahissant l'espace parapharyngé à droite

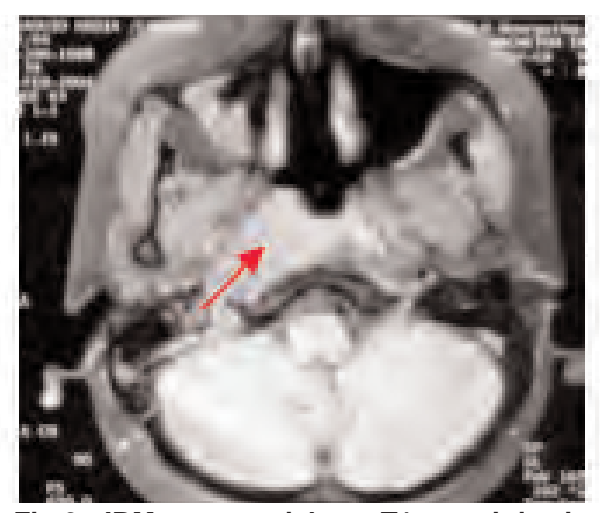

Fig.2 : IRM coupe axiale en T1 avec injection

de gadolinium : Prise de contraste de façon hétérogène d'une tumeur de la paroi latéral du cavum

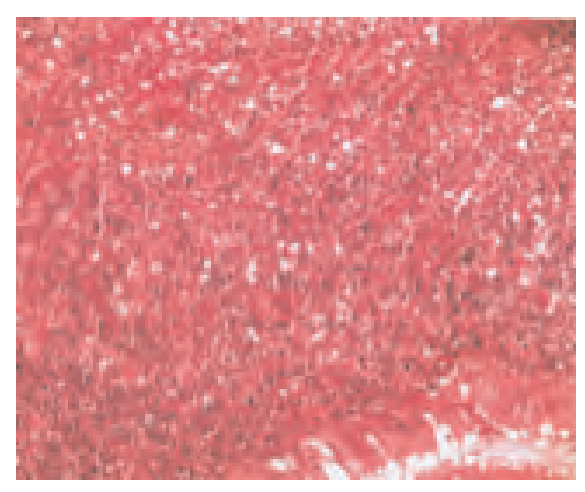

Fig.3 : (HE x 200) Infiltration de la muqueuse nasopharyngée par des plage de cellules ronde à noyau macronucléolé évoquant un mélanome malin

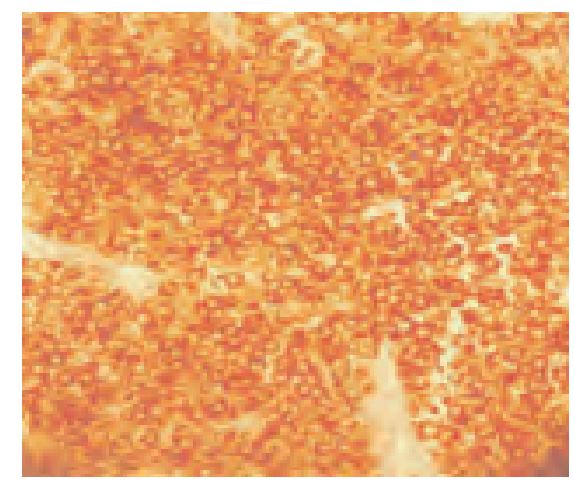

Fig.4 : Immuno marquage positif pour la protéine $\mathrm{S} 100$ évoquant un mélanome malin

Observation 2 :

Madame AR, âgée de 37 ans, a été hospitalisée en juin 2003 pour dysphagie haute aux solides, céphalées, épistaxis à répétition et hypoacousie gauche. L'examen endoscopique révélait une tumeur bourgeonnante intéressant la paroi latérale gauche du nasopharynx, s'étendant en bas au niveau de la paroi pharyngée latérale jusqu'à la limite supérieure du sinus piriforme gauche. II n'y avait 
pas d'adénopathies cervicales.

La TDM et I'IRM montrent une extension à la base du crâne ( Fig. 5 et 6). La biopsie du cavum était en faveur d'un carcinome mucoépidermoïde de haut grade (fig 7 et 8).

Après bilan d'extension locorégional, la tumeur était classée T4NOM0 (UICC 97) (extension de la base du crâne jusqu'à l'hypopharynx et du cavum vers la fosse infratemporale).

La patiente a été traitée par une chimio-radiothérapie concomitante à la dose de 64 Gray sur le cavum et les aires ganglionnaires. Actuellement, après 17 mois de recul, la patiente est en rémission complète locale et à distance.

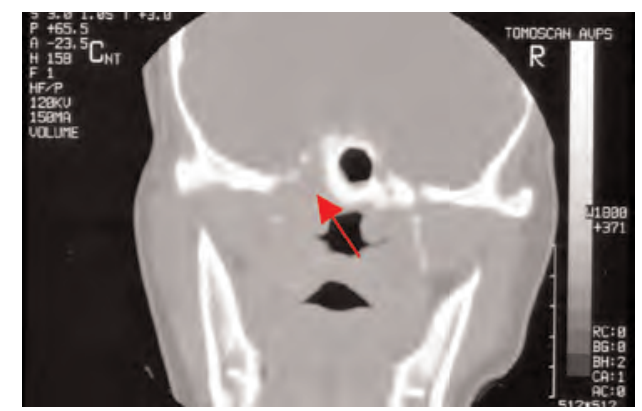

Fig 5 : TDM en coupe coronale : extension vers la base du crâne d'une tumeur du cavum

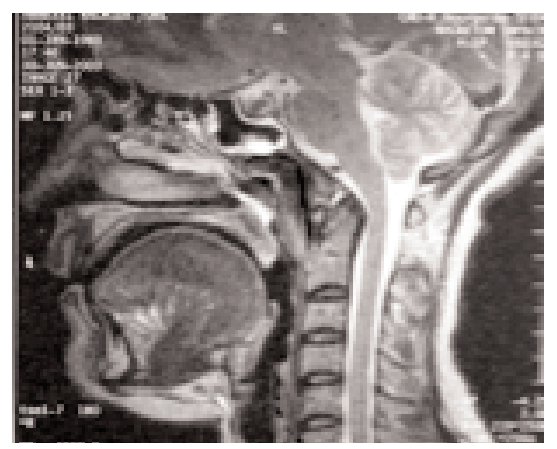

Fig.6 : IRM coupe sagittale T2 : extension vers la base du crâne d'une tumeur du cavum

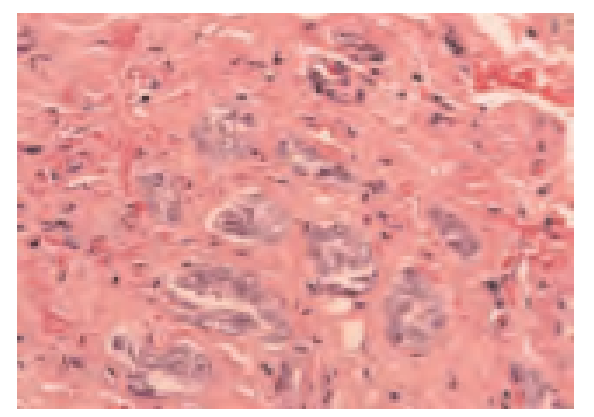

Fig.7 : Coupe histologique de la biopsie du cavum montrant des noyaux cellulaires en ilots arrondis évoquant une composante glandulaire

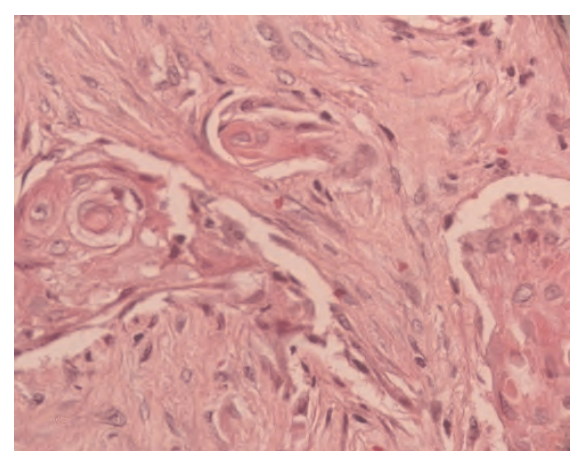

Fig.8 : Coupe histologique de la biopsie du cavum montrant la composante malpighienne de la tumeur, qui associée à l'aspect de la figure 7 pose le diagnostic de carcinome mucoépidermoide.

\section{Observation 3 :}

Madame R, âgée de 33 ans a consulté pour une hypoacousie et céphalée hémicranienne droite évoluant depuis 7 mois. L'examen a montré un bombement de la paroi latérale droite du cavum et de la face postérieure du palais mou avec refoulement en bas et en dedans de l'amygdale droite sans adénopathies cervicales palpables.

L'examen anatomopathologique de la biopsie du cavum a conclu à un carcinome adénoïde kystique type cribrifome sans invasion périnerveuse ( fig 9).

Le scanner du cavum a montré une masse tumorale de la paroi latérale du cavum avec envahissement parapharyngé ( fig 10). A l'IRM, il s'agissait d'un processus expansif intéressant la paroi latérale droite du cavum et l'espace préstylien droit de $4,5 \mathrm{~cm}$ de grand axe en hypersignal T2, se rehaussant de façon intense après injection de Gadolinium et indépendant du lobe profond de la parotide (fig.11, 12).

Le bilan d'extension était normal (une radiographie du thorax, échographie abdominale et scintigraphie osseuse).

Le diagnostic de carcinome adénoïde kystique du cavum (T2bN0M0) (UICC 97) a été retenu. La patiente a été opérée par voie transmandibulaire médiane avec exérèse tumorale incomplète autour de l'axe vasculaire suivi d'un évidement ganglionnaire triangulaire homolatéral.

Une RT externe postopératoire à la dose de 50 Gray a été réalisée. Un an après, la patiente a présenté des métastases pulmonaires pour lesquelles elle a eu 4 cures de chimiothérapie entraînant une régression partielle des métastases. Huit mois après la fin du traitement, elle a eu une grossesse qu'elle n'a pas voulu interrompre. La grossesse a été menée à terme sans anomalies foetales. Actuellement à 18 mois de recul, elle est en rémission locale clinique et radiologique avec persistance des métastases pulmonaires. (Fig. 13) 


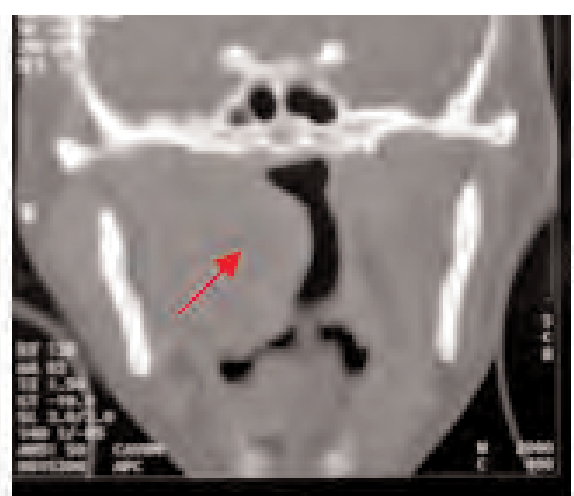

Fig.10 : TDM coupe coronale : énorme tumeur de la paroi latérale droite du cavun arrivant à la base du crane sans l'envahir

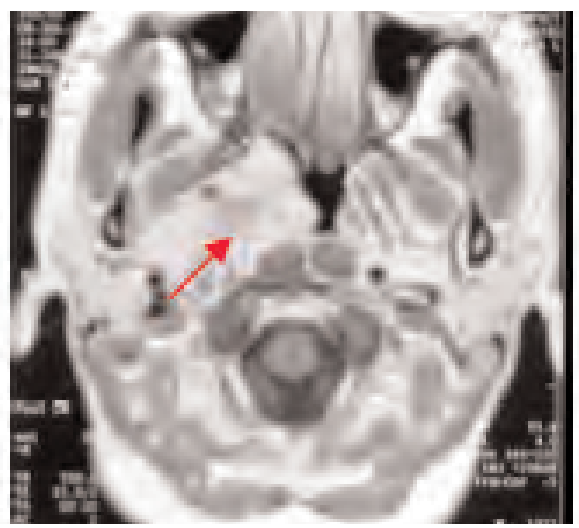

Fig.11 : IRM coupe axiale T1 post gadolinium : la tumeur est en hypersignal hétérogène, envahissant l'espace parapharyngé et reste indépendante du lobe profond de la parotide

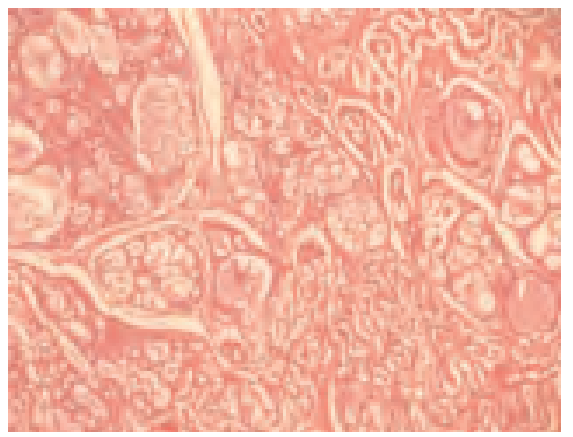

Fig.12 : Coupe histologique : disposition des noyaux cellulaires en structure tubulaire bien adossée évoquant un cylindrome de type cribriforme

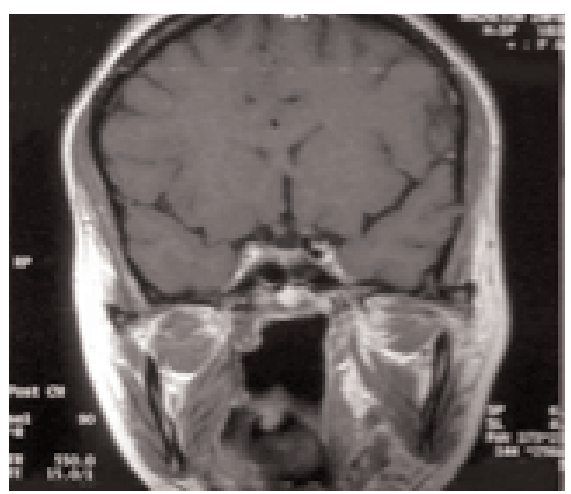

Fig.13 : IRM (post opératoire) coupe coronale en T1 post gadolinium :

disparition totale de la tumeur.

\section{COMMENTAIRES}

Les mélanomes malins primitifs (MMP) des muqueuses cervico-faciales sont rares. Ils représentent 0.8 à $2 \%$ de l'ensemble des mélanomes de l'organisme (1). La localisation rhinopharyngée est exceptionnelle (2). Plusieurs théories ont été proposées pour expliquer l'histogénèse de MMP du cavum sans qu'aucune ne soit retenue (3). Le traitement des MMP se base, arbitrairement et en l'absence d'une classification et d'une conduite internationale codifiée, sur la chirurgie $(4,5)$. Cependant, la localisation profonde du cavum et la découverte de tumeurs à des stades avancés, fait que la chirurgie des MM du cavum est souvent délabrante sans être radicale (5). Un curage ganglionnaire, selon la plupart des auteurs, peut être proposé en présence d'adénopathies palpables $(4,5)$. Le second traitement pouvant être utilisé devant un MMP du cavum est la radiothérapie. Cependant, ces tumeurs sont connues radio-résistantes (6). L'utilisation de la chimiothérapie devant les MM est appuyée sur la fréquence des métastases à distance. Elle se base surtout sur le déticène ou le cis-platinum. Cependant aucun produit n'a prouvé son efficacité devant les MMP quelque soit sa localisation $(2,4)$.

Actuellement certaines études sont en cours pour évaluer l'effet de l'interféron et de l'interleukine2 (IL2) sur le MMP $(7,8)$. En effet, la constatation de certains cas de régression spontanée de MMP a fait évoquer une origine immunologique de ces tumeurs. Le pronostic du MMP reste réservé avec une survie de 1 à 60 mois (5).

Le carcinome mucoépidermoïde (CME) est une entité rare représentant 5 à $10 \%$ des tumeurs des glandes salivaires $(8,9,10)$.

La classification adoptée est celle de l'OMS 1991 révisée en 2004 et qui reconnaît seulement deux grades: CME de bas grade et de haut grade. Pour la localisation nasopharyngée, le traitement de choix est similaire à celui du CME des glandes salivaires accessoires qui associe une exérèse chirurgicale combinée à la radiothérapie. Ce traitement permet en général d'améliorer le pronostic (8). 
En tenant compte du taux élevé de métastases ganglionnaires cervicales occultes, il est recommandé de compléter par un curage cervical bilatéral (8). Chez notre patiente, la tumeur était étendue à tout le pharynx, arrivant en haut jusqu'à la base du crâne et envahissant latéralement la fosse infra-temporale. La chirurgie serait alors délabrante et dans tous les cas incomplète.

L'évolution clinique et le pronostic dépendent du stade clinique et du grading histologique: les CME de haut grade sont caractérisés par un comportement évolutif plus agressif avec un risque de récidive et de métastase ganglionnaires élevé. Paradoxalement, ces formes de haut grade présentent un faible potentiel métastatique hématogène $(8,9,10)$.

Les carcinomes adénoïdes kystiques sont des tumeurs épithéliales malignes se développant au dépens des glandes salivaires principales et surtout accessoires (11, $12,13)$. La localisation nasopharyngée est rare (14).

La chirurgie première avec radiothérapie (RT) postopérartoire constitue le traitement de choix des tumeurs malignes des glandes salivaires accessoires (11, 15, $14,16,17)$. Cependant l'exérèse totale peut se heurter à des difficultés liées à la localisation anatomique du nasopharynx, et à la nature infiltrante et extensive de ce type de tumeur ce qui expose au risque de récidive locale même en cas d'exérèse macroscopiquement complète. L'attitude vis-à-vis des aires ganglionnaires n'est pas encore codifiée. Certains sont partisans pour un évidement ganglionnaire étant donné que la présence de métastases ganglionnaires serait cause d'échec thérapeutique $(11,12,13,15)$.
La RT postopératoire permet d'améliorer le taux de contrôle local en cas d'exérèse incomplète des tumeurs avancées avec supériorité de la neutronthérapie permettant un taux de survie à 5 ans égal à $77 \%$ contre 60 à 75 $\%$ pour la RT conventionnelle.

La place de la chimiothérapie (CT) est controversée. Pour certains auteurs, elle a permis d'obtenir des réponses partielles ou parfois complètes. Pour d'autres, aucune réponse n'a été notée. La polychimiothérapie à base de Cisplatine, d'Adriamycine, ou de 5-Fluorouracile serait plus efficace $(12,14)$.

Le pronostic dépend essentiellement de la qualité d'exérèse et du type histologique du carcinome adénoïde kystique. En effet, une exérèse complète avec des limites histologiques saines offrent un pronostic meilleur $(16,18$, 19).

Sur le plan histologique, le type solide est de pronostic défavorable avec un taux de survie à 15 ans de l'ordre de $5 \%$ contre $39 \%$ pour les tumeurs sans composante solide. Pour l'invasion périnerveuse, certains auteurs l'ont considérée comme élément de mauvais pronostic (16). Pour d'autres, seule la présence d'un envahissement nerveux macroscopique serait pourvoyeuse de récidives locales et à distance. Pour notre patiente, il n'y avait pas d'atteinte des nerfs crâniens et à l'histologie, pas d'invasion périnerveuse.

\section{REFERENCES}

1- Stoll D., Boudard P., Deminière C. Mélanome malin primitif des muqueuses ORL. Etude à propos de 5 cas. Rev Laryngol Otol Rhinol 1989; 110: 229-31. 2- Berthelsen A, Andersen AP, Jensen S, Hansen HS. Melanomas of the mucosa in the oral cavity and the upper respiratory passages. Cancer 1984; 54: 90712.

3- Ram M.D. Apudomas. Current Surg. 1981; 38: 230-3.

4- Baltchford SJ, Koopmann CF, Coulthard SW. Mucosal melanoma of the head and neck. Laryngoscope 1986; 96:929-34.

5- Enée V., Houliat T., Truilhé Y., Darrouzet V., Stoll D. Mélanomes malins des muqueuses naso-sinusiennes. Etude rétrospective à propos de 20 cas. Rev Laryngol Otol Rhinol 2000; 121:243-50.

6- Pande Y.N. Malignant melanoma of the post nasal space. J Laryngol Otol 1970; 84:1065-7.

7- James G. et Al. Treatment of locally advanced adenoïd cystic carcinoma of the head and neck with neutron radiotherapy. International Journal of radiation oncology biology physics. 2000; 3: 551-7.

8- Lund V. Malignant melanoma of the nasal cavity and paranasal sinuses. J Laryngol Otol 1982; 96: 347-55.

9 - Brown J et al. Nasopharyngectomy for recurrent high grade mucoépidermoïd carcinoma after radiation failure. Otolaryngal head and Neck Surg 1999; 120 (4): 564-6.

10- $\mathrm{He} \mathrm{JH}$ et al. Clinicopathological characteristics of primary nasopharyngeal adénocarcinoma. Al Zheng 2003; 22 (7): 753-7.

11- Simoncelli C., Altissimi G., Tassoni A. A propos du cylindrome cervicofacial.
Revue de laryngologie 1984;105 (2); 79-87.

12- Manipoud P., Manipoud J.P., Kemeny J.L., Leclercq L. Carcinome adénoïde kystique : à propos d'une localisation au sac lacrymal. Ann Otolaryngol Chir Cervicofac. 1994; 111: 295-8.

13- Victor L., Schramm J.r., Mario J., Imola, M. Management of nasopharyngeal salivary gland malignancy. Laryngoscope. 2001; 111: 1533-44.

14- Saâdi I. et al. Carcinome adénoïde kystique du nasopharynx : à propos d'un cas. Cancer-Radiothérapie: 2003; 190: 4.

15- Dai Takagi and al. Clinical study of adenoid cystic carcinoma of the head and neck. Auris Nasus larynx. 2001; 28: 99-102.

16- Chummun S., Mclean N.R., Kelly C.G., et al. Adenoid cystic carcinoma of the head and neck. British journal of plastic surgery. 2001; 54: 476-48.

17- Truilhe Y., Richaud P., Houliat T. et al. Place de la chirurgie dans le traitement des cancers du cavum classés T4. Evaluation préliminaire à partir d'une série de 18 cas. Ann Otolaryngol Chir Cervicofac. 2002; 119(3) : 138-45.

18- Adam S, Garden M.D, Randal S.et Al. The influence of positive margins and nerve invasion in adenoid cystic carcinoma of the head and neck treated with surgery and radiation. Int. J Radiotion oncolory Biol. Phys. 1995; 32: 619-26. 19- James G. et Al. Treatment of locally advanced adenoïd cystic carcinoma of the head and neck with neutron radiotherapy. International Journal of radiation oncology biology physics. 2000; 3: 551-7. 